\title{
Dye lasing in optically manipulated liquid aerosols
}

\author{
Yasin Karadag ${ }^{a}$, Mehdi Aas ${ }^{a}$, Alexandr Jonáš ${ }^{a}$, Suman Anand ${ }^{b}$, David McGloin ${ }^{b}$, and Alper \\ $\mathrm{Kiraz}^{a, c}$ \\ ${ }^{a}$ Department of Physics, Koç University, Rumelifeneri Yolu, Sariyer, 34450 Istanbul, Turkey; \\ ${ }^{b}$ Electronic Engineering and Physics Division, University of Dundee, Nethergate, Dundee DD1 \\ $4 \mathrm{HN}, \mathrm{UK}$ \\ ${ }^{c}$ Koç University TÜPRAŞ Energy Center (KUTEM), Koç University, Rumelifeneri Yolu, \\ Sariyer, 34450 Istanbul, Turkey
}

\begin{abstract}
We present dye lasing from optically manipulated glycerol-water aerosols with diameters ranging between 7.7 and $11.0 \mu \mathrm{m}$ confined in optical tweezers. While being optically trapped near the focal point of an infrared laser, the droplets stained with Rhodamine B were pumped with a Q-switched green laser and their fluorescence emission spectra featuring whispering gallery modes (WGMs) were recorded with a spectrograph. Nonlinear dependence of the intensity of the droplet WGMs on the pump laser fluence indicates dye lasing. The average wavelength of the lasing WGMs could be tuned between 600 and $630 \mathrm{~nm}$ by adjusting the droplet size. These results may lead to new ways of probing airborne particles, exploiting the high sensitivity of stimulated emission to small perturbations in the droplet laser cavity and the gain medium.
\end{abstract}

Keywords: Aerosol, Nonlinear Optics, Lasing, Optical Trapping

\section{INTRODUCTION}

Due to their perfectly spherical shape, micron-sized liquid droplets can serve as natural optical resonant cavities hosting high-quality whispering gallery modes (WGMs). Enhanced optical fields and small modal volumes of such WGMs enable observation of non-linear optical phenomena at pump power thresholds that are significantly lower than those required for bulk samples. Precise characterization of the droplet size and material properties via cavity-enhanced Raman spectroscopy ${ }^{1}$ and building of microscopic lasers based on liquid cavities containing a gain medium ${ }^{2}$ are possible because of the ease of triggering the nonlinear optical response of droplets. Microlasers based on liquid cavities have already found applications in biological and chemical sensing. ${ }^{3,4}$ Attractiveness of such optofluidic microlasers is further enhanced by the possibility of controlled tuning of their emission spectral characteristics by adjusting the shape, size, and composition of liquid microcavities . ${ }^{5,6}$

First successful demonstration of lasing in airborne microdroplets dates back almost thirty years ago when lasing was observed in freely flowing streams of droplets. ${ }^{7}$ However, due to the lack of position stabilization and control, freely moving liquid aerosols have only a limited use in practical applications in sensing and microlaser design. The stabilization of droplet position over extended time periods is necessary to fully exploit the potential of lasing WGMs in microdroplets. Depositing droplets of water and other polar liquids on a superhydrophobic surface can be an effective way for the position stabilization. ${ }^{8}$ Active, non-invasive control of the droplet position by external micromanipulation then represents the next step in the practical exploitation of lasing aerosols. To date, lasing from airborne microdroplets has been demonstrated in electrodynamic ${ }^{9}$ and acoustic $^{10}$ trapping configurations. Optical tweezing is another micromanipulation technique that is especially well suited for stable confinement of very small droplets and readily adaptable for simultaneous manipulation of large numbers of particles. ${ }^{11}$ Various studies employed optical tweezing to localize liquid aerosols over long periods of time for applications in a large variety of fields including atmospheric chemistry and physics, and health science. ${ }^{12,13}$

Further author information: (Send correspondence to A.J., A.K. and D.M.)

A.J.: E-mail: ajonas@ku.edu.tr, Telephone: +90 2123381588

A.K.: E-mail: akiraz@ku.edu.tr, Telephone: +90 2123381701

D.M.: E-mail: d.mcgloin@dundee.ac.uk, Telephone: +44 (0) 1382384402

Optical Trapping and Optical Micromanipulation X, edited by Kishan Dholakia, Gabriel C. Spalding, Proc. of SPIE Vol. 8810, 88100P - (c) 2013 SPIE · CCC code: 0277-786X/13/\$18 · doi: 10.1117/12.2024166 
Optical tweezing has also been used to confine and move solid lasing microspheres ${ }^{14}$ or lasing emulsion droplets. ${ }^{15}$ However, up to now lasing from liquid aerosols manipulated using optical tweezers has not been demonstrated.

In this work, we demonstrate lasing from individual optically trapped liquid aerosol particles doped with a fluorescent dye and optically pumped with a focused pulsed laser beam. We characterize the droplet emission spectrum as a function of the droplet size and demonstrate that the droplet lasing wavelength can be tuned over an interval larger than $30 \mathrm{~nm}$ by changing the droplet radius. In addition, we study the dynamics of the droplet lasing spectra that provides information about the changes of the droplet size due to liquid evaporation/condensation and coalescence of individual trapped droplets.

\section{EXPERIMENTAL SETUP}

Optical setup used for the experiments with trapped aerosol lasing was described previously ${ }^{15}$ and is shown in Fig. 1. A continuous wave solid state infrared laser $(\lambda=1064 \mathrm{~nm}, 300 \mathrm{~mW}$ maximum power; Crystalaser $)$ was used for aerosol trapping. The trapping laser beam was sent through a beam expander and focused into the sample chamber by a water immersion microscope objective $(\mathrm{NA}=1.2,60 \mathrm{x}$; Nikon) in the inverted microscope geometry.

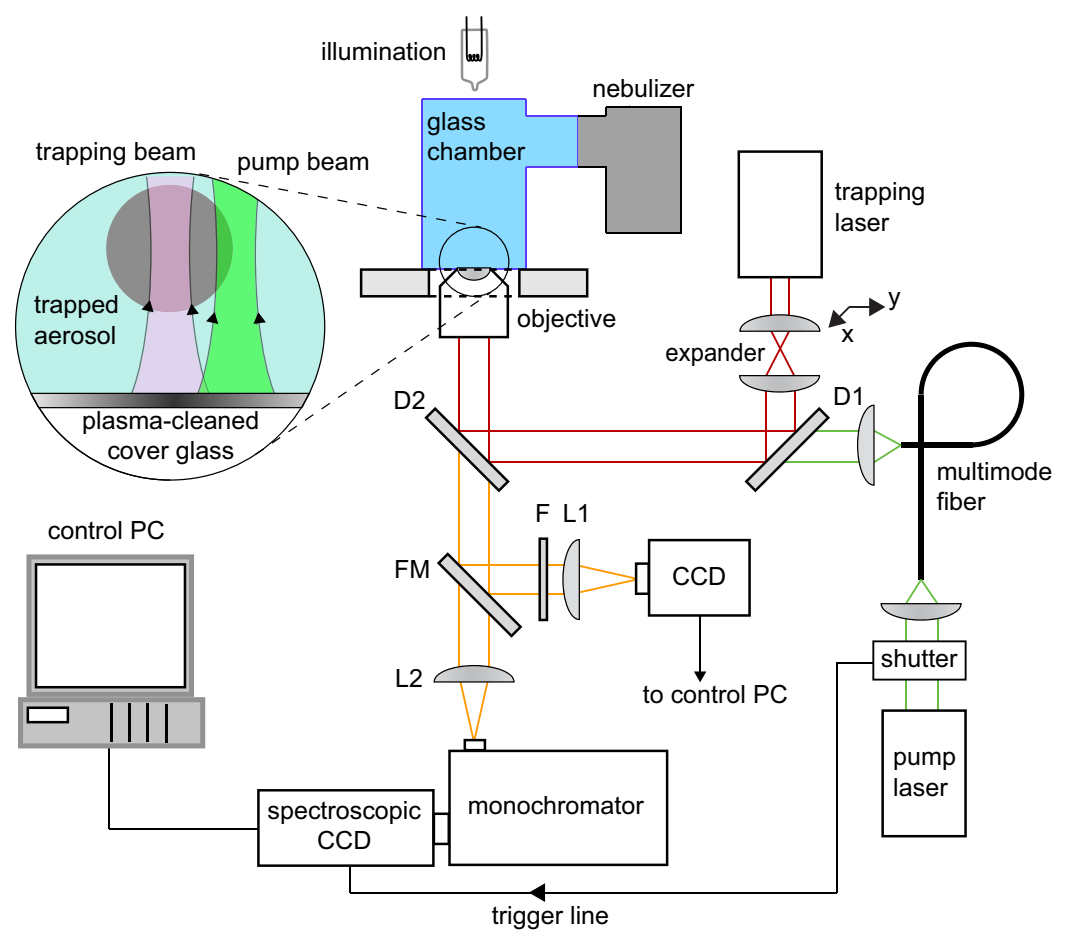

Figure 1. Experimental setup for studies of lasing in optically trapped aerosols. The inset shows the detail of an optically trapped aerosol particle with the pump laser beam positioned on the particle rim. D1, D2 - dichroic mirrors, F - band-pass filter, FM - flippable mirror, L1, L2 - lenses.

Based on the previous work, ${ }^{13}$ the power of the trapping beam was set to $\sim 3.5 \mathrm{~mW}$ at the focus of the microscope objective for stable on-axis trapping of aerosols with diameters ranging between 5-10 $\mu$ m. Pulsed green beam $(\lambda=532 \mathrm{~nm}, 20 \mathrm{~ns}$ pulse width and $33 \mathrm{kHz}$ repetition rate) obtained after frequency-doubling the output of a home-built, passively Q-switched $\mathrm{Nd}: \mathrm{YVO}_{4}$ laser was used for pumping the trapped microdroplets. The pump beam was delivered to the setup through a multi-mode fiber, combined with the trapping beam by a dichroic mirror, and focused by the same microscope objective that was employed for optical trapping to a $9.8 \mu \mathrm{m}$ diameter spot. In our experiments, the trapping and pump beams were focused at the same plane approximately $20 \mu \mathrm{m}$ above the upper surface of the cover glass that formed the bottom part of the sample chamber. For efficient excitation of WGMs in the trapped droplet by on-edge optical pumping, the focal spot 
of the pump beam was approximately centered at the rim of the droplet. The fluorescence emission from the droplets was collected using the same microscope objective and dispersed by a monochromator (focal length 500 mm; Acton Research) on the chip of a cooled CCD detector (Pixis 100; Princeton Instruments). In order to prevent fast photobleaching of the dye molecules in the droplets, a shutter was added to the pump laser beam path. This shutter was synchronized with the CCD detector and triggered the spectrum acquisition during an exposure time of $10 \mathrm{~ms}$.

A compact ultrasonic nebulizer (JIH50; Beuer) was used for generating liquid aerosols out of 39\% w/w glycerol/water solution (refractive index $n_{\text {int }}=1.38$, density $\rho=1.099 \mathrm{~g} / \mathrm{cm}^{3}$ ) doped with $1 \mathrm{mM}$ Rhodamine B dye. The nebulizer was attached to a closed glass sample chamber that prevented external air flow from disturbing the stability of the trapped aerosol particles and maintained approximately constant ambient humidity during the experiments. A cover glass treated with $\mathrm{Ar} / \mathrm{O}_{2}$ plasma was attached at the bottom of the sample chamber. Plasma cleaning of cover glasses ensured a good hydrophilicity of their surface, thus preventing accumulation of large sessile droplets that otherwise disturb the trapping and pump beams focused into the chamber. During the experiments, initial diameters of the trapped droplets were generally around $5 \mu \mathrm{m}$. Larger droplets were then obtained by coalescence of the trapped droplets with others that were attracted to the trapping region. Trapped droplet diameters $d$ were estimated from microscope images and independently determined from the observed WGM free-spectral range (FSR), mean lasing wavelength $\bar{\lambda}$, and known refractive indices of the droplet $\left(n_{\text {int }}=1.38\right)$ and air $\left(n_{\text {ext }}=1.00\right)$, using the asymptotic formula of Chylek et al.: ${ }^{16}$

$$
d=\frac{\bar{\lambda}^{2}}{\pi n_{\mathrm{ext}} \mathrm{FSR}} \frac{\arctan \left(\left[\left(n_{\mathrm{int}} / n_{\mathrm{ext}}\right)^{2}-1\right]^{1 / 2}\right)}{\left[\left(n_{\mathrm{int}} / n_{\mathrm{ext}}\right)^{2}-1\right]^{1 / 2}} .
$$

\section{RESULTS}

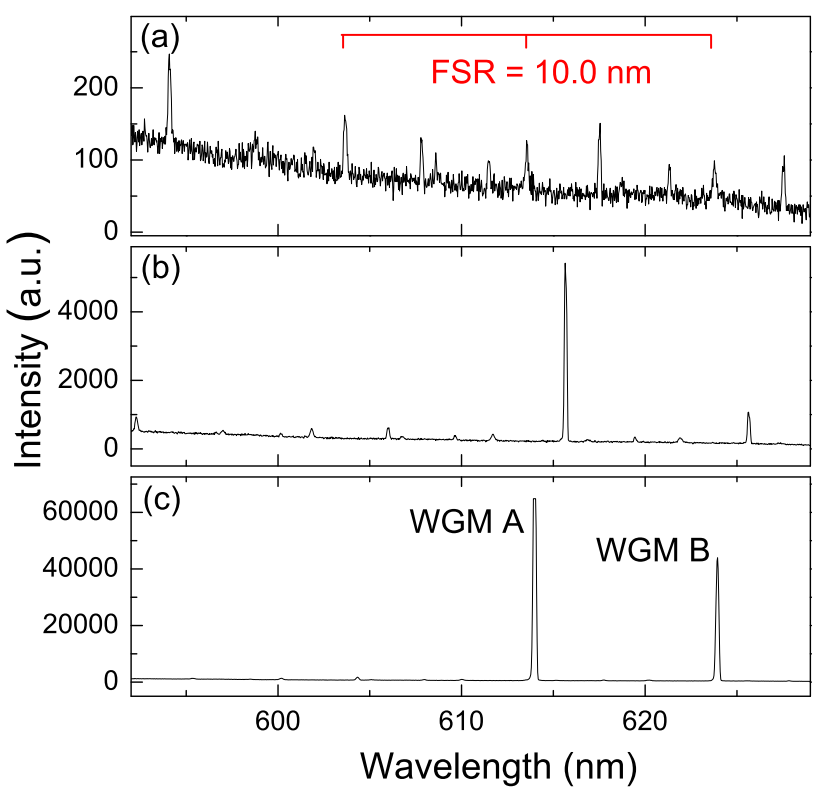

Figure 2. Excitation fluence-dependent emission spectra recorded from a $9.2 \mu \mathrm{m}$ diameter glycerol/water droplet at three different excitation fluences: (a) $0.27 \mathrm{~mJ} / \mathrm{cm}^{2}$, (b) $1.04 \mathrm{~mJ} / \mathrm{cm}^{2}$, and (c) $2.4 \mathrm{~mJ} / \mathrm{cm}^{2}$. WGM A and WGM B denote lasing WGMs.

Fig. 2 shows fluorescence emission spectra recorded from a trapped $9.2 \mu \mathrm{m}$ diameter glycerol/water aerosol droplet at varying excitation fluences. Non-lasing WGMs of the aerosol particle were observed in Fig. 2(a) when the excitation fluence was lower than the lasing threshold. These WGMs are superimposed on a broad non-resonant background emission from the dye-doped thin solution layer formed on the cover glass during the 
experiment. In general, droplet WGMs can be characterized using radial, azimuthal, and angular mode numbers, and polarization (TE or TM). ${ }^{6}$ The WGMs that belong to the same mode family with identical radial mode number and polarization are indicated in Fig. 2(a). Spectral separation between two such consecutive WGMs gives the FSR which is equal to $10.0 \mathrm{~nm}$ for the droplet studied in Fig. 2. In order to observe lasing, the cavity losses must be smaller than the net cavity gain. Excitation fluence and the amount of dye in the aerosol particle are critical to satisfy this condition. As shown in Fig. 2(b), two WGMs denoted as A and B exhibit lasing around $\lambda=615 \mathrm{~nm}$ and $\lambda=625 \mathrm{~nm}$ upon crossing the threshold excitation fluence of $0.28 \mathrm{~mJ} / \mathrm{cm}^{2}$. The spectral positions of lasing WGMs display a small residual drift $\Delta \lambda=3.5 \mathrm{~nm}$ over the total duration of the experiment $(2 \mathrm{~min})$. For a droplet with the mean diameter of $9.2 \mu \mathrm{m}$, this translates into the drift of the droplet diameter by $\Delta d=\Delta \lambda(d / \lambda)=52.8 \mathrm{~nm}$ caused by evaporation of the aqueous content of the droplet.

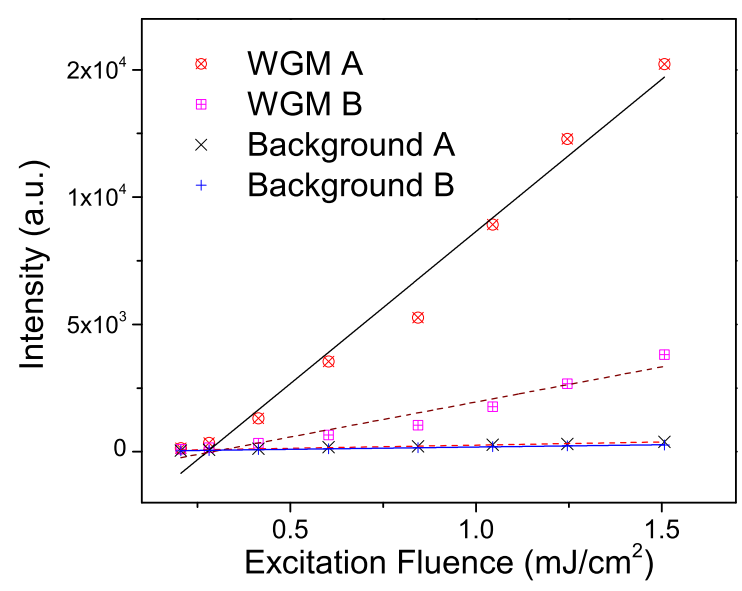

Figure 3. Power-dependent intensities of the lasing WGMs and background emissions in a $9.2 \mu$ m diameter glycerol/water aerosol droplet.

Fig. 3 displays the intensities of the lasing WGMs A and B indicated in Fig. 2 and non-resonant spectral background in the vicinity of these WGMs as a function of the excitation fluence. Both lasing and background intensities were determined from Lorentzian fits to the selected WGMs in a series of emission spectra acquired with gradually increasing excitation fluence. Subsequently, two lines were fitted to the respective lasing and background intensity series and the intersection of the fitted lines provided the threshold excitation fluence of $0.28 \mathrm{~mJ} / \mathrm{cm}^{2}$ for both WGM A and WGM B. ${ }^{15}$ As expected, the background intensity increased linearly with the excitation fluence for all values of fluence within the studied range; this also indicated that the photobleaching effects during the acquisition of the excitation fluence-dependent spectral series could be neglected owing to the short exposure time required for recording individual spectra. When the excitation fluence exceeded the threshold, increase of the intensity of the lasing peak with a higher slope relative to the background emission indicated the onset of lasing. The threshold excitation fluence required for the observation of lasing depends on the size of the trapped aerosol particle and the Q-factor of its WGMs. ${ }^{17}$ Large radiative losses in smaller droplets result in an exponential decrease of their WGM Q-factors. ${ }^{18}$ This radiative loss sets a lower limit to the diameter of droplets that display lasing at a given maximal excitation fluence. In our experimental conditions, this minimal droplet diameter was determined to be $7.7 \mu \mathrm{m}$.

With increasing droplet size, spectral position of the emission maximum of lasing aerosols containing constant dye concentration was observed to shift to longer wavelengths. This trend is illustrated in Fig. 4(a) which shows emission spectra of six droplets with different diameters recorded at fixed $2.41 \mathrm{~mJ} / \mathrm{cm}^{2}$ excitation fluence. Here, the increasing droplet diameter corresponds to the decreasing FSR of the droplet (see Eq. 1). For the given droplet, spectral location of its overall maximal lasing gain can be quantitatively characterized by the mean lasing wavelength $\bar{\lambda}$ calculated as the intensity-weighted average of the wavelengths of all lasing WGMs from the same mode family observed in the droplet's spectrum. Fig. 4(b) summarizes the relationship between $\bar{\lambda}$ and FSR for 34 lasing droplets of varied size. Within the studied range of droplet sizes (7.7 $\mu \mathrm{m}$ to $11.0 \mu \mathrm{m})$, FSR 


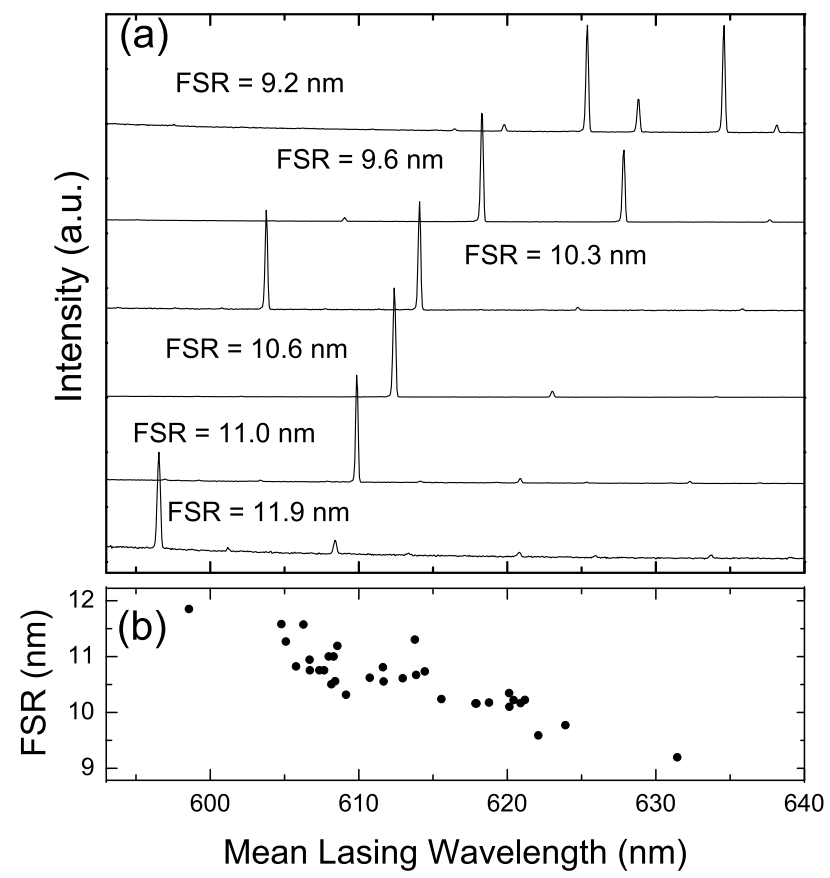

Figure 4. (a) Lasing spectra recorded from 6 different aerosols with diameters 7.7, 8.4, 9.0, 9.6, 10.1, and 11.0 $\mu$ m (bottom to top) at constant excitation fluence of $2.41 \mathrm{~mJ} / \mathrm{cm}^{2}$. (b) Free spectral ranges of 34 lasing aerosols as a function of the mean lasing wavelength, $\bar{\lambda}$.

decreased with $\bar{\lambda}$ following an almost linear dependence. A similar spectral shift of the overall lasing gain profile was previously observed in lasing emulsion droplets ${ }^{6,15}$ and explained by the dependence of the efficiency of light out-coupling from the droplets and the effective cross-section of the lasing WGMs on the droplet size.
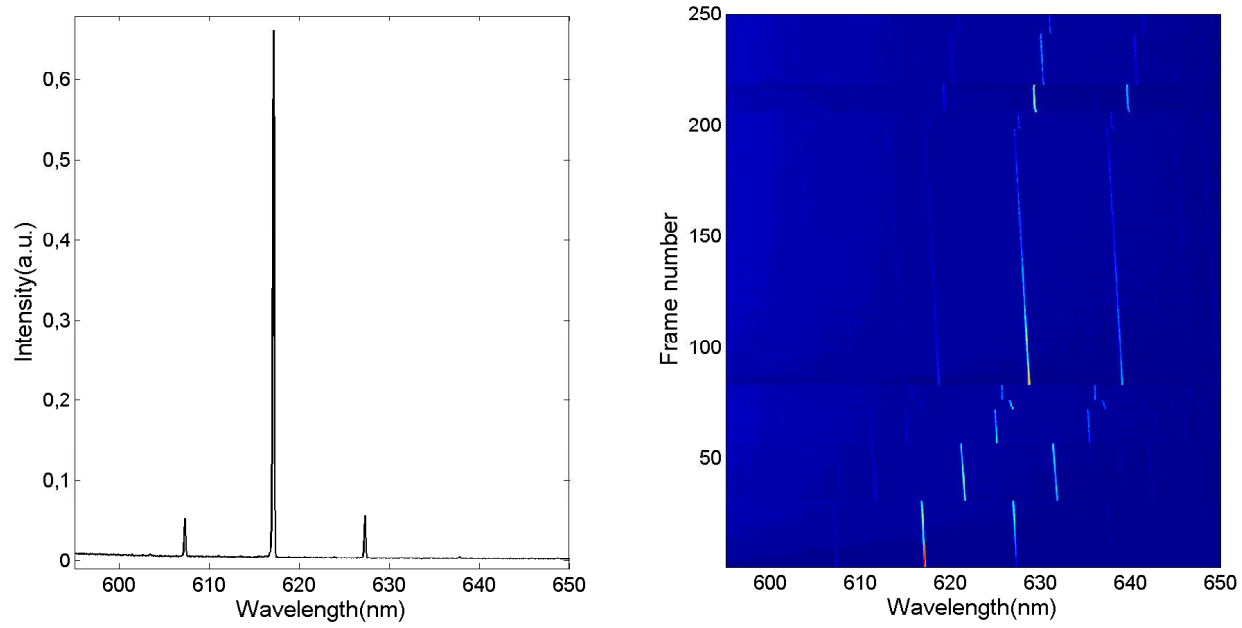

Figure 5. (a)Lasing emission spectrum of a $9.2 \mu \mathrm{m}$ diameter aerosol acquired immediately after the droplet confinement in the optical trap. (b)2D plot of consecutive lasing emission spectra recorded from same aerosol as in (a).

During confinement in the optical trap, droplet size generally changes as a result of a complex interplay between liquid evaporation induced by laser heating and condensation in the environment with high relative humidity set by the bulk glycerol/water solution. Such changes of the droplet size are sensitively reflected in the positions of the lasing WGMs. Figure 5a shows the lasing emission spectrum of a trapped $9.2 \mu$ m diameter 
aerosol acquired shortly after the droplet confinement in the trap. Figure 5b then shows consecutive lasing emission spectra recorded from the same trapped aerosol. Time interval between individual spectral acquisitions was $20 \mathrm{~ms}$. From this figure, it is obvious that the lasing WGMs gradually drifted to the blue end of the spectrum, indicating decrease in the droplet size due to water evaporation. As reported in Refs. ${ }^{6,15}$ this heating-induced evaporation resulted mainly from the dye absorption. In addition to the slow blue drift, sudden red shifts of the lasing WGM positions occured at random times. Such instantaneous spectral changes were a direct consequence of coalescence of the trapped aerosol with other droplets floating in the sample chamber. Since the resulting coalesced droplet had a bigger size, the lasing WGMs shifted to longer wavelengths.

\section{CONCLUSIONS}

We have demonstrated lasing in the WGMs of dye-doped glycerol/water microdroplets manipulated in air using optical tweezers. By changing the trapped droplet size, we have shown that the average lasing wavelength could be tuned between 600-630 nm. Controlled fusion of multiple droplets confined in dynamically positioned independent optical traps, manipulation of relative humidity and temperature in the sample chamber, or local heating with the trapping laser can be further used for precise control of the lasing droplet size and, thus, emission wavelength tuning (see Fig. 5 for illustration). Our results are readily adaptable to simultaneous manipulation of large numbers of lasing aerosol particles. They also bring up the possibility of using the high sensitivity of stimulated emission to small perturbations in the shape, size, and material properties of the droplet cavity for sensitive chemical and biological analysis in airborne particles.

\section{ACKNOWLEDGMENTS}

This work was partially supported by TÜBİTAK (Grant No. 111T059). S.A. thanks the Schlumberger Faculty for the Future program for support.

\section{REFERENCES}

[1] Reid, J. P., Meresman, H., Mitchem, L., and Symes, R., "Spectroscopic studies of the size and composition of single aerosol droplets," Int. Rev. Phys. Chem. 26, 139-192 (2007).

[2] Mølhave, K., Kristensen, A., and Mortensen, N. A., [Advanced Photonic Structures for Biological and Chemical Detection], ch. Droplet-Based Cavities and Lasers, 471-486, Springer (2009).

[3] Tanyeri, M. and Kennedy, I. M., "Detecting single bacterial cells through optical resonances in microdroplets," Sensor Lett. 6, 326-329 (2008).

[4] Humar, M. and Musevic, I., "Surfactant sensing based on whispering-gallery-mode lasing in liquid-crystal microdroplets," Opt. Express 19, 19836-19844 (2011).

[5] Humar, M., Ravnik, M., Pajk, S., and Muševič, I., "Electrically tunable liquid crystal optical microresonators," Nature Photonics 3, 595-600 (2009).

[6] Tang, S. K. Y., Derda, R., Quan, Q., Loncar, M., and Whitesides, G. M., "Continuously tunable microdroplet-laser in a microfluidic channel," Opt. Express 19, 2204-2215 (2011).

[7] Tzeng, H.-M., Wall, K. F., Long, M. B., and Chang, R. K., "Laser emission from individual droplets at wavelengths corresponding to morphology-dependent resonances," Opt. Lett. 9, 499-501 (1984).

[8] Kiraz, A., Sennaroglu, A., Doganay, S., Dundar, M. A., Kurt, A., Kalaycoglu, H., and Demirel, A. L., "Lasing from single, stationary, dye-doped glycerol/water microdroplets located on a superhydrophobic surface," Opt. Commun. 276, 145-148 (2007).

[9] Tona, M. and Kimura, M., "Novel lasing modes observed in a levitated single dye-doped microdroplet," Journal of the Physical Society of Japan 69, 3533-3535 (2000).

[10] Azzouz, H., Alkhafadiji, L., Balslev, S., Johansson, J., Mortensen, N. A., Nilsson, S., and Kristensen, A., "Levitated droplet dye laser," Opt. Express 14, 4374-4379 (2006).

[11] Burnham, D. R. and McGloin, D., "Holographic optical trapping of aerosol droplets," Optics Express 14, 4176-4182 (2006).

[12] Hopkins, R. J., Mitchem, L., Ward, A. D., and Reid, J. P., "Control and characterisation of a single aerosol droplet in a single-beam gradient-force optical trap," Phys. Chem. Chem. Phys. 6, 4924 (2004). 
[13] McGloin, D., Burnham, D. R., Summers, M. D., Rudd, D., Dewar, N., and Anand, S., "Optical manipulation of airborne particles: techniques and applications," Faraday Discuss. 137, 335-350 (2008).

[14] Sasaki, K., Fujiwara, H., and Masuhara, H., "Photon tunneling from an optically manipulated microsphere to a surface by lasing spectral analysis," Appl. Phys. Lett. 70, 2647 (1997).

[15] Aas, M., Jonáš, A., and Kiraz, A., "Lasing in optically manipulated, dye-doped emulsion microdroplets," Opt. Commun. 290, 183-187 (2013).

[16] Chylek, P., Kiehl, J. T., and Ko, M. K. W., "Optical levitation and partial-wave resonances," Phys. Rev. A 18, 2229 (1978).

[17] Campillo, A. J., Eversole, J. D., and Lin, H. B., "Cavity quantum electrodynamic enhancement of stimulated emission in microdroplets," Phys. Rev. Lett. 67, 437-440 (1991).

[18] Serpengüzel, A., Swindal, J. C., Chang, R. K., and Acker, W. P., "Two-dimensional imaging of sprays with fluorescence, lasing, and stimulated raman scattering," Appl. Opt. 31, 3543-3551 (1992). 